\title{
Lack of antigenic diversification of major outer membrane proteins during clonal waves of Neisseria meningitidis serogroup A colonization and disease
}

\author{
Charlotte A. Huber ${ }^{1,2}$, Valentin Pflüger ${ }^{1,2}$, Abdul-Wahab M. Hamid ${ }^{3}$, Abudulai A. Forgor ${ }^{3}$, Abraham Hodgson $^{3}$,
} Ali Sié ${ }^{4}$, Thomas Junghanss ${ }^{5} \&$ Gerd Pluschke ${ }^{1,2}$

1 Swiss Tropical and Public Health Institute, Basel, Switzerland

2 University of Basel, Basel, Switzerland

3 Navrongo Health Research Centre, Ministry of Health, Navrongo, Ghana

4 Centre de Recherche en Sante de Nouna, Nouna, Burkina Faso

5 Section of Clinical Tropical Medicine, University Hospital Heidelberg, Heidelberg, Germany

This very interesting study compares outer membrane protein sequences in a number of Neisseria meningitidis serogroup. A disease and carriage isolates from a defined region or subsaharan Africa between 2002 and 2008. It concludes that there is a remarkable antigenic stability in this population and that herd immunity is not a strong driving force for antigenic diversification. The study is well conceived and carried out, the results are clearly presented and the conclusions pertinent. This is important and useful information

\section{Keywords}

Neisseria meningitidis; outer membrane protein; herd immunity.

\section{Correspondence}

Charlotte A Huber, University of Queensland Centre for Clinical Research, Level 8, Building 71/918 Royal Brisbane Hospital, Herston, Qld 4006, Australia.

Tel.: (61) 733466073

fax: (61) 733465598

e-mail: c.huber@uq.edu.au

Received: 16 January 2012; revised 17 August 2012; accepted 21 August 2012. Final version published online 28 November 2012.

doi:10.1111/2049-632X.12000

Editor: Jacques Schrenzel

\begin{abstract}
In particular in the 'meningitis belt' of sub-Saharan Africa, epidemic meningococcal meningitis is a severe public health problem. In the past decades, serogroup $A$ lineages have been the dominant etiologic agents, but also other serogroups have caused outbreaks. A comprehensive vaccine based on subcapsular outer membrane proteins (OMPs) is not available. Here, we have investigated whether meningococcal populations overcome herd immunity by changing antigenic properties of their OMPs. Meningococcal isolates were collected in the context of longitudinal studies in Ghana between 2002 and 2008 and in Burkina Faso between 2006 and 2007. Serogroup A strains isolated during two clonal waves of colonization and disease showed no diversification in the genes encoding their PorA, PorB, and FetA proteins. However, we detected occasional allelic exchange of opa genes, as well as wide variation in the number of intragenic tandem repeats, showing that phase variation of Opa protein expression is a frequent event. Altogether we observed a remarkable antigenic stability of the PorA, PorB and FetA proteins over years. Our results indicate that while herd immunity may be responsible for the disappearance of meningococcal clones over time, it is not a strong driving force for antigenic diversification of the major OMPs analyzed here.
\end{abstract}

\section{Introduction}

Neisseria meningitidis, a Gram-negative diplococcus, is an obligate commensal of humans, usually carried in the upper airways of healthy individuals. The bacterium is transmitted to close contacts by the spread of respiratory secretions. Occasionally, $N$. meningitidis strains might penetrate the mucosal membrane, multiply in the bloodstream, and gain access to the cerebrospinal fluid. The overall incidence rate of meningococcal disease in Europe and North America is 1-3 per 100000 person-years. The highest incidence rates are found in countries of sub-Saharan Africa, in the so-called 'meningitis belt', which extends from Ethiopia to Senegal. In these countries, annual incidence rates of as high as 1000 per 100000 person-years are recorded during the most severe epidemics (Caugant, 2008). The savannah climate of the meningitis belt with an annual precipitation index of $300-1100 \mathrm{~mm}$ and extremely dry and hot winter seasons seems to be optimal for transmission of invasive meningococcal disease. Epidemics classically occur in the dry season, between December and April. They nearly always start in the early part of the dry season and then stop abruptly at the onset of rains. High temperature, low absolute humidity and the harmattan (a dusty wind that 
blows from the Sahara) at the end of the dry season may favor the occurrence of meningococcal disease by damaging the local mucosal defenses (Greenwood, 1999).

Based on different capsular polysaccharide structures, 13 serogroups of $N$. meningitidis can be distinguished. The vast majority of invasive meningococcal disease is caused by six of these serogroups ( $A, B, C, W-135, X$, and $Y$ ) (Stephens et al., 2007). Most of the large epidemics in Africa in the past 100 years were associated with serogroup A (Caugant, 2008). Until recently, reactive immunization campaigns with polysaccharide vaccines initiated after the onset of meningitis epidemics have been used to limit epidemics. Now a monovalent conjugate vaccine against serogroup A meningococci, the most common cause of large epidemics, is being introduced stepwise in the countries of the African meningitis belt. In contrast to the unconjugated polysaccharides, conjugate vaccines will not only protect the vaccinated from invasive disease, but are also expected to block transmission of the meningococci expressing the vaccine serogroup.

Lack of a stable nasopharyngeal population of apathogenic meningococci may contribute to the vulnerability of the local population of the meningitis belt to major epidemics (Leimkugel et al., 2007b). These are caused by hypervirulent clones of $N$. meningitidis, which may travel through the entire meningitis belt, but seem to remain only for a few years associated with a particular population (Leimkugel et al., 2007a, b). Herd immunity may lead to the disappearance of individual clonal complexes, but new clones with a different antigenic make-up may subsequently spread. Clonal waves of colonization and disease are therefore a characteristic feature of the epidemiology of meningococcal meningitis in the African meningitis belt (Leimkugel et al., $2007 a$, b). Neisseria meningitidis serogroup A strains of the clonal complex 5 that are associated with the sequence types (STs) 5, 7, and 2859 have been responsible for outbreaks in the last two decades (Nicolas et al., 2005; Teyssou \& Muros-Le, 2007; Sie et al., 2008). Molecular typing approaches based on DNA sequencing allow meningococci to be distinguished and tracked (Jolley et al., 2007). During the last decade, multi-locus sequence typing (MLST) based on the identification of the alleles of fragments of seven meningococcal housekeeping genes has developed into the 'gold standard' for typing these bacterial pathogens (Maiden et al., 1998; Brehony et al., 2007).

Meningococci are naturally competent for transformation by exogenous DNA, and high rates of recombination have been observed (Jolley et al., 2005). However, most genetic exchange appears to takes place between closely related meningococci, and it has been suggested that recombination may be primarily a mechanism for genome repair that will only occasionally result in generation of diversity (Caugant \& Maiden, 2009). Nevertheless, N. meningitidis is genetically and antigenically highly diverse (http://pub $\mathrm{mlst}$.org/neisseria/). On the other hand, hyper-invasive lineages seem to be surprisingly stable over decades and during global spread (Caugant, 2008). This may facilitate development of a comprehensive protein-based vaccine, effective against a broad range of hyper-virulent meningococci. Cross-reactivity could be achieved by targeting antigenically invariant subcapsular structures or by combining a cocktail of vaccine antigens selected on the basis of molecular epidemiological studies. A number of meningococcal surface structures are thought to play a role in mucosal colonization, hematogenous spread and crossing of the blood brain barrier (Hill et al., 2010). To escape immune surveillance, meningococci have developed a range of mechanisms to change surface components. We assume that development of herd immunity is responsible for the complete disappearance of meningococcal clones after a few years of colonization of populations in the African meningitis belt (Leimkugel et al., 2007a, b). Here we have investigated whether meningococcal populations escape from immune detection by varying their outer membrane proteins (OMPs) PorA, PorB, FetA, and Opa. The serogroup A ST7 and ST 2859 meningococci analyzed have been collected between March 2002 and April 2008 in the course of longitudinal meningococcal colonization and disease surveys in Ghana and Burkina Faso (Leimkugel et al., 2007a, b; Sie et al., 2008).

\section{Materials and methods}

\section{Bacterial isolates}

The $N$. meningitidis isolates investigated in this study had been collected in the Kassena-Nankana District (KND) and the neighboring district of Bawku in Ghana and in the Nouna Health District (NHD) in the Kossi region of Burkina Faso. Case strains were isolated from the cerebrospinal fluid of meningitis patients, and carriage strains were isolated from throat swabs collected in the context of longitudinal carriage surveys. Isolation and characterization of strains has been described previously (Gagneux, 2000; Leimkugel et al., 2007a, b; Sie et al., 2008). For the analysis of genetic diversification, serogroup A ST7 and ST2859 strains isolated from cases and carriers at different times during clonal colonization and disease waves were selected from our strain collection. Included were nine ST7 carriage and seven ST7 case isolates collected between March 2002 and March 2005 in the KND of Ghana. In the case of ST2859 strains, we analyzed six case and seven carriage isolates collected between March 2006 and March 2007 in the NHD of Burkina Faso, three case and 15 carriage isolates from the KND, as well as three case isolates collected between March 2007 and April 2008 in the neighboring district of Bawku.

\section{Genetic analysis}

DNA was extracted from bacterial pellets using the Wizard ${ }^{\mathbb{R}}$ Genomic DNA Purification Kit (Promega AG, Duebendorf, Switzerland). The DNA concentration was measured using a Nano drop Spectrophotometer (Witec Ag, Littau, Switzerland). PCR was performed using $5 \mu \mathrm{L}$ of $10 \times \mathrm{BD}$ buffer and $1 \mu \mathrm{L}$ of FirePol Taq polymerase, $1.25 \mathrm{mM} \mathrm{MgCl}_{2}$ (Solis BioDyne, Tartu, Estonia), $100 \mathrm{ng}$ of genomic DNA or the equivalent volume of nuclease-free water as a negative 
control, a $0.2 \mu \mathrm{M}$ concentration of each forward and reverse primer, and a $0.2 \mathrm{mM}$ concentration of each deoxynucleoside triphosphate in a total volume of $50 \mu \mathrm{L}$. PCRs were run in a T Professional Basic PCR machine (Biometra $\mathrm{GmbH}$, Göttingen, Germany). The thermal profile for PCR amplification included an initial denaturation step of $94^{\circ} \mathrm{C}$ for $2 \mathrm{~min}$, followed by 35 cycles of $94^{\circ} \mathrm{C}$ for $30 \mathrm{~s}$, annealing at $55^{\circ} \mathrm{C}$ for $30 \mathrm{~s}$, and elongation at $72{ }^{\circ} \mathrm{C}$ for $1 \mathrm{~min} 20 \mathrm{~s}$ up to $2 \mathrm{~min}$. The PCRs were finalized by an extension step at $72{ }^{\circ} \mathrm{C}$ for $10 \mathrm{~min}$. PCR products were analyzed on $1 \%$ agarose gels by gel electrophoresis using ethidium bromide staining and the Alphalmager illuminator and Alphalmager software (Alpha Innotech, San Leandro, CA). PCR products were purified using a NucleoSpin purification kit (Macherey-Nagel $\mathrm{GmbH} \&$ Co. KG, Düren, Germany) and subjected to direct sequencing or cloned using a TOPO TA cloning kit (Invitrogen Corp., Carlsbad, CA), transformed into Novablue competent cells (Merck, Darmstadt, Germany), and sequenced after DNA preparation (Macherey-Nagel GmbH \& Co. KG, Düren, Germany). Sequencing was performed by Macrogen Inc, Seoul, Korea. For PorA, PorB, and FetA typing, primers were used as previously described (Sacchi et al., 1998; Thompson et al., 2003; Russell et al., 2004). Primers used for amplification and sequencing of the opa genes and their flanking regions are listed in Table 1.

Design of the primers used for amplification of opaA, opaB, and opaD was based on the serogroup A strain Z2491 genome sequence (Parkhill et al., 2000) and the PRIMER3 program (http://frodo.wi.mit.edu/). The sequences obtained were analyzed making use of the $N$. meningitidis homepage (http://neisseria.org/nm/) and the Neisseria sequence typing homepage (http://pubmlst.org/neisseria/). The multiple sequence alignment websites Multalin (http://bioinfo.genopoletoulouse.prd.fr/multalin/multalin.html) and CLUSTALW2 (http:// www.ebi.ac.uk/Tools/clustalw2/index.html) were used for comparisons between Opa gene sequences and their flanking regions. The EMBOSS TRANSEQ tool was used for the translation of the retrieved opa gene sequences (http://www.ebi.ac.uk/Tools/ emboss/transeq/).

\section{Results and discussion}

Humans are the only natural host for $N$. meningitidis, and nasopharyngeal carriage rates are known to be much higher than disease rates. In a longitudinal study in the northern Ghana, we have observed waves of colonization and disease with hyper-virulent clones of serogroup A meningococci. About 4 years after their local emergence, these clones disappeared completely (Leimkugel et al., 2007a, b). This may be attributed to the development of herd immunity against the colonizing clone in the local population. During their spread from country to country, meningococcal clones undergo microevolution with recombination as major driving force for genetic variation (Achtman, 1995). As a consequence, clones that, based on MLST, appear to be closely related, may differ - due to multiple recombination events -

Table 1 Amplification and sequencing primers for opa genes and their flanking regions

\begin{tabular}{|c|c|c|c|}
\hline \multirow[t]{6}{*}{ OpaA } & Amplification primers & VNTR15af & TCATCCGCTACATTGTGTTGA \\
\hline & & OpaA2r & TCGTCATTCCCACGGAAGT \\
\hline & Sequencing primers & VNTR15af & TCATCCGCTACATTGTGTTGA \\
\hline & & OpaA4r & TTTCCTGATTTTCCGTCTTCA \\
\hline & & OpaA5r & ATGACGGTTCGGGTATTTCC \\
\hline & & OpaA4f & GCGGCAGATTATGCCAGTTA \\
\hline \multirow[t]{6}{*}{ OpaB } & Amplification primers & OpaB2f & CAGGACAAGGCGACGAG \\
\hline & & OpaB5r & TGTCTGGACGGGGATGT \\
\hline & Sequencing primers & VNTR15br & GCACACCGATATAGGGTTTGAA \\
\hline & & OpaBf & GTGTTGAAACATCGCCACAA \\
\hline & & OpaBr1 & GGCATTTTTCCATGCGTTT \\
\hline & & OpaBff & GCGAGAACTGAAGACGGAAA \\
\hline \multirow[t]{7}{*}{ OpaD } & Amplification primers & OpaD5f & TCTCCGTAGAGGAAATGATGC \\
\hline & & OpaD3r & AAGTGGGAATCTAGGACGTAAAA \\
\hline & Sequencing primers & OpDf & TCATCCGCTATATTGTGTTGA \\
\hline & & Opa26f & TGGGTCTTGGTGTCATCG \\
\hline & & OpA26r & GAATAATTACTTTCTTTCCATTTTCTG \\
\hline & & OpD2f & CGCCCCAAACCTGATATAGT \\
\hline & & OpDr2 & GAAACGGTGGGAATTGTGTAA \\
\hline \multirow[t]{8}{*}{ OpaJ } & Amplification primers & Opaj5f & CGCCCCAAACCTGATATAGT \\
\hline & & Opaj1r & ATCTAGAACGTGGGGTTTGG \\
\hline & Sequencing primers & Opaj5f & CGCCCCAAACCTGATATAGT \\
\hline & & Opaj7f & TGATATAGTCCGCTCCTGCAA \\
\hline & & Opaj8f & CGGTGCAGACAAAGACAAAA \\
\hline & & Opaj9f & GTCGCCGGTGCTGCTA \\
\hline & & Opaj10r & TAGCAGCACCGGCGAC \\
\hline & & Opaj3r & TTTGGGCAACTGTTTTTATCC \\
\hline
\end{tabular}




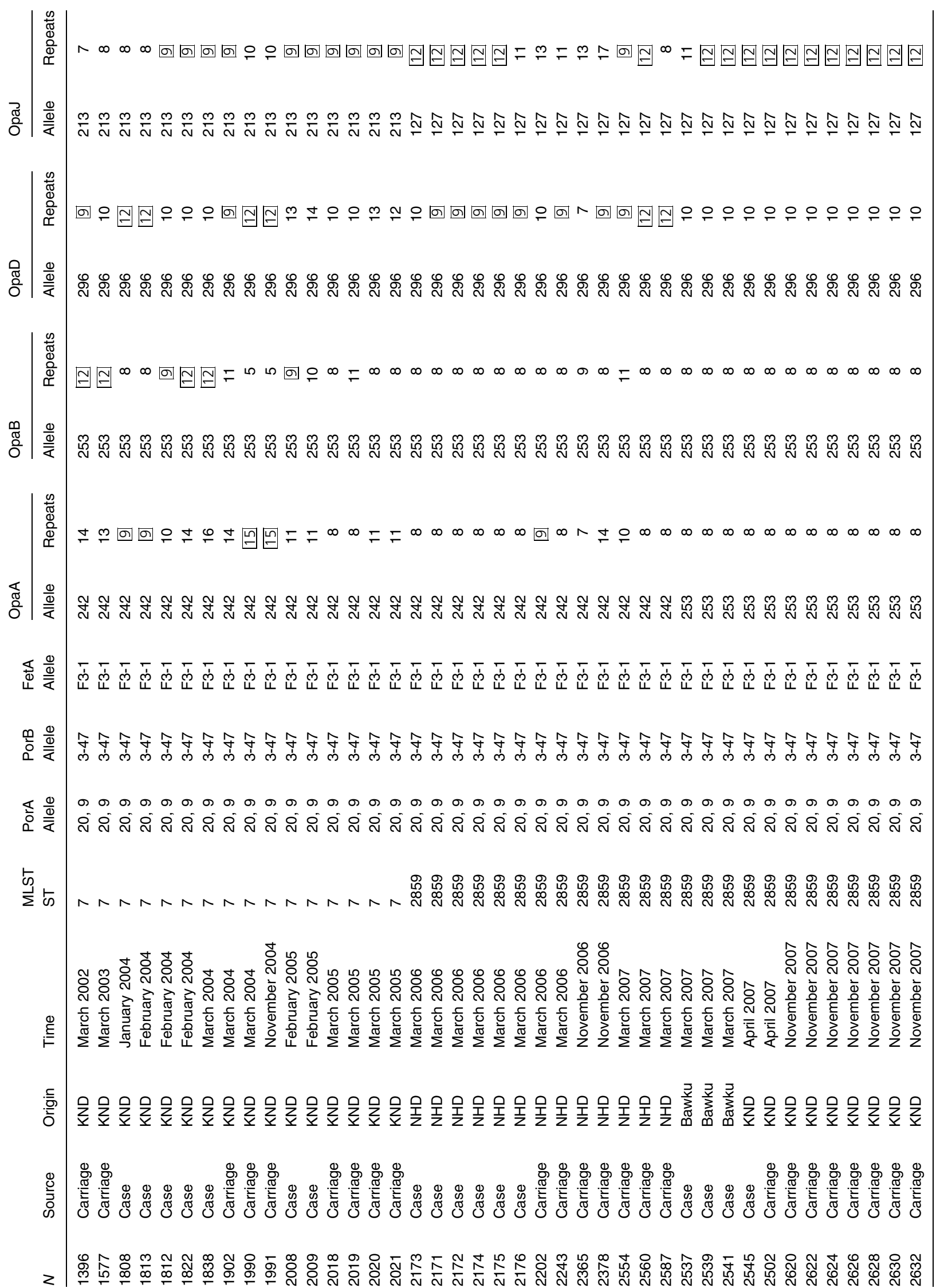




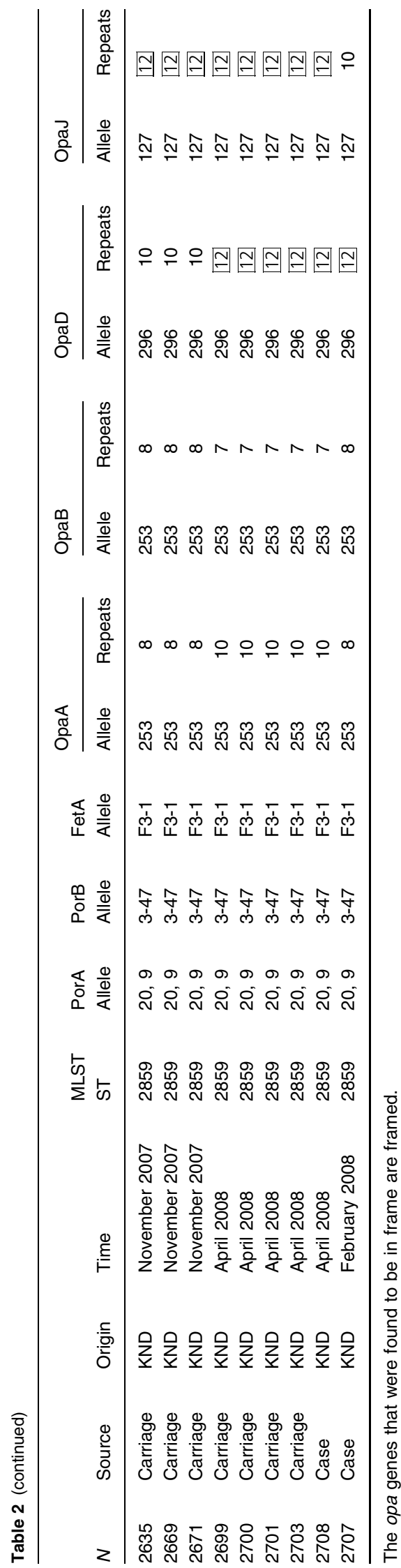

very substantially in their antigenic profiles $A$. Lamelas and G. Pluschke, Swiss Tropical and Public Health Institute.

Antibodies directed against OMPs have been implicated in the development of natural immunity against meningococci. In particular, antibodies directed against the PorA and PorB proteins seem to provide serosubtype-specific protection (Jordens et al., 2004). This prompted us to investigate whether immune selection pressure building up in the population during colonization waves leads to an antigenic diversification of OMPs in the colonizing meningococcal population.

The strains included in the sequence analysis of OMPencoding genes have been isolated from cerebrospinal fluid (CSF) of meningitis patients or from the pharynx of healthy carriers during two sequential colonization and disease waves in northern Ghana and during a meningococcal disease outbreak in Burkina Faso. Serogroup A ST7 strains have been isolated from the start (2002) till the end (2005) of a colonization wave in northern Ghana (Leimkugel et al., $2007 a, b)$. In the case of serogroup A ST 2859, isolates from an outbreak in Burkina Faso (2006-2007) (Sie et al., 2008) and in northern Ghana (2007-2008) were compared.

It has been proposed that the propensity of $N$. meningitidis to accumulate mutations increases dramatically toward the end of an epidemic, presumably due to immune pressure, and that with time, variants arise that are founders of new clonal complexes (Achtman, 2004). However, in none of the strains investigated here a single mutational change in the genes encoding the surface proteins PorA, PorB, or FetA was found. All PorA sequences had the VR1, VR2: 20,9 variable regions. The Por $B$ sequences were invariably of class 3 and had the allele 47 . The variable region of Fet A was F3-1. In spite of microevolution of the epidemic clones, as detected by pulsed field gel electrophoresis (Leimkugel et al., 2007a, b), the sequence of the analyzed OMP-encoding genes thus was strikingly stable. No mutations were detected in the variable regions of the surface proteins PorA, PorB, and FetA in any of the strains analyzed. The benefit of accumulating mutations to escape herd immunity may be dispossessed by the fitness cost that such mutations confer (Achtman, 2004). Furthermore, the lack of a genetically diverse pharyngeal flora of $N$. meningitidis, in the study population (Leimkugel et al., 2007a, b), limiting the extent of horizontal genetic exchange (Achtman, 1995) may contribute to this striking lack of diversification. On the other hand, immune selection may act more strongly on exposed antigens other than the major OMPs analyzed here.

While the Ghanian meningococcal isolates analyzed here have been collected over a longer period of time (between 2002 and 2008), conclusions on the stability of OMPs for the strains from Burkina Faso are less strong, as the analyzed strains were isolated over a period of only 2 years. Another very important point to consider is that, although we did not detect any diversification in the genes encoding PorA, Por B, and FetA, expression levels of PorA and FetA are subject to variation. Antibodies elicited against these proteins in the host population may give selective advantage to meningococcal strains with decreased expression levels 
of PorA and FetA (Crowe et al., 1989; Carson et al., 2000). PorB expression levels have been described to be constant, however (Abad et al., 2006).

The multiple copies of genes encoding the Opa proteins can be turned on and off during chromosomal replication by slipped-strand mispairing of tandem CTTCT repeats present in their open reading frames. Here, we have used the published genome sequence of the serogroup A ST4 strain Z2491 to design primers specific for the flanking regions of the opaA, opaB, and opaD genes. In contrast to strain Z2491, the ST7 and ST2859 strains analyzed here all contained in addition an opaJ gene, as has been described for other ST5-complex/subgroup III strains (Callaghan et al., 2006). While all PorA, PorB, and FetA had the same alleles irrespective of the time point and location of isolation as well as the ST of the strains examined (Table 2), two of the four opa genes analyzed were subject to some variation. While all the ST7 strains from Ghana and the ST2859 strains from Burkina Faso had the opaA allele 242, all Ghanaian ST2859 strains had the allele 253. No variation was observed within the three individual groups of epidemiologically related isolates. ST7 and ST2859 are MLST single-locus variants, and the ST2859 genocloud may have developed from ST7 meningococci in Africa. The change in the opaA allele in the ST 2859 strains that emerged in Ghana in 2008 may have been due to homologous recombination and immune selection against the 242 allele (Callaghan et al., 2008; Levin \& Cornejo, 2009). In the case of the opaJ gene, all ST7 isolates had the allele 213, whereas the ST2859 strains both from Ghana and from Burkina Faso had the allele 127.

The numbers of pentanucleotide repeats within the coding sequences of the opa genes were subject to much wider variation. However, strains isolated at the same location and a similar time point tended to be relatively uniform. For example, all Ghanaian ST2859 isolates from 2007 had 8 opaA, 8 opaB, and 10 opaD repeats, while $5 / 6$ isolates from 2008 had 10 opaA, 7 opaB, and 12 opaD repeats. This variation in numbers of tandem repeats is due to slippedstrand mispairing and results in phase variation involving on/ off expression of the respective opa gene (Murphy et al., 1989). Alleles with a number of pentanucleotide repeats are a multiple of three represent functional genes (Table 2). Antigenic variation in the Opa genes has been suggested to mediate immune evasion (Davidsen \& Tonjum, 2006; Callaghan et al., 2008). Functional open reading frames were found for all four opa genes, but no more than two functional opa genes were found in any of the strains. A Poisson regression analysis was performed (http://www. fisherstat.com/Pages/default.aspx), and disease and colonization isolates did not differ significantly in the Opa protein expression patterns (Table 2). While the invasive bacteria may represent rare genetic variants of the colonizing meningococcal population, a change in the Opa protein expression pattern thus does not seem to be relevant for an adaptation for multiplication in the blood stream and the CSF.

Control of meningitis epidemics has relied so far on reactive vaccination strategies with polysaccharide vaccines, and a serogroup A conjugate vaccine is currently being introduced in the meningitis belt. In our study, we have observed an apparent lack of diversification of PorA, PorB, and FetA, and a tailor-made vaccine targeting those OMPs may be able to control the epidemic strains investigated. However, to develop a comprehensive vaccine based on subcapsular OMPs, whole-genome analyses of epidemiologically welldefined isolate collections may be needed. Comparative genomics may give insight into the driving forces behind the microevolution of $N$. meningitidis, allowing a more comprehensive analysis of antigen variability, as well as the identification of new vaccine targets. Results of such analyses may thus help identifying antigens suitable for inclusion into a multivalent protein subunit vaccine (Tan et al., 2010).

\section{Acknowledgements}

This work was supported by the Volkswagen Foundation through the Collaborative Research Grant (SFB) 544 'Control of Tropical Infectious Diseases'. This publication made use of the Neisseria Multi-Locus Sequence Typing website (http://pubmlst.org/neisseria/) developed by Keith Jolley and Man-Suen Chan (Jolley et al., 2004, BMC Bioinformatics, 5: 86) and sited at the University of Oxford. The development of this site has been funded by the Wellcome Trust and European Union.

\section{References}

Abad R, Alcala B, Salcedo C, Enriquez R, Uria MJ, Diez $P$ \& Vazquez JA (2006) Sequencing of the porB gene: a step toward a true characterization of Neisseria meningitidis. Clin Vaccine Immunol 13: 1087-1091.

Achtman M (1995) Epidemic spread and antigenic variability of Neisseria meningitidis. Trends Microbiol 3: 186-192.

Achtman M (2004) Population structure of pathogenic bacteria revisited. Int J Med Microbiol 294: 67-73.

Brehony C, Jolley KA \& Maiden MC (2007) Multilocus sequence typing for global surveillance of meningococcal disease. FEMS Microbiol Rev 31: 15-26.

Callaghan MJ, Jolley KA \& Maiden MC (2006) Opacity-associated adhesin repertoire in hyperinvasive Neisseria meningitidis. Infect Immun 74: 5085-5094.

Callaghan MJ, Buckee CO, Jolley KA, Kriz P, Maiden MC \& Gupta $S$ (2008) The effect of immune selection on the structure of the meningococcal opa protein repertoire. PLOS Pathog 4: e1000020.

Carson SD, Stone B, Beucher M, Fu J \& Sparling PF (2000) Phase variation of the gonococcal siderophore receptor FetA. Mol Microbiol 36: 585-593.

Caugant DA (2008) Genetics and evolution of Neisseria meningitidis: importance for the epidemiology of meningococcal disease. Infect Genet Evol 8: 558-565.

Caugant DA \& Maiden MC (2009) Meningococcal carriage and disease-population biology and evolution. Vaccine 27(suppl 2): B64-B70.

Crowe BA, Wall RA, Kusecek B, Neumann B, Olyhoek T, Abdillahi $\mathrm{H}$, Hassan-King M Greenwood BM, Poolman JT \& Achtman M (1989) Clonal and variable properties of Neisseria meningitidis isolated from cases and carriers during and after an epidemic in The Gambia, West Africa. J Infect Dis 159: 686-700. 
Davidsen T \& Tonjum T (2006) Meningococcal genome dynamics. Nat Rev Microbiol 4: 11-22.

Gagneux S (2000) Microheterogeneity of serogroup A (subgroup III) Neisseria meningitidis during an outbreak in northern ghana. Trop Med Int Health 5: 280-287.

Greenwood B (1999) Manson lecture. Meningococcal meningitis in Africa. Trans R Soc Trop Med Hyg 93: 341-353.

Hill DJ, Griffiths NJ, Borodina E \& Virji M (2010) Cellular and molecular biology of Neisseria meningitidis colonization and invasive disease. Clin Sci 118: 547-564.

Jolley KA, Wilson DJ, Kriz P, McVean G \& Maiden MC (2005) The influence of mutation, recombination, population history, and selection on patterns of genetic diversity in Neisseria meningitidis. Mol Biol Evol 22: 562-569.

Jolley KA, Brehony C \& Maiden MC (2007) Molecular typing of meningococci: recommendations for target choice and nomenclature. FEMS Microbiol Rev 31: 89-96.

Jordens JZ, Williams JN, Jones GR, Christodoulides M \& Heckels JE (2004) Development of immunity to serogroup B meningococci during carriage of Neisseria meningitidis in a cohort of university students. Infect Immun 72: 6503-6510.

Leimkugel J, Forgor AA, Dangy JP, Pfluger V, Gagneux S, Hodgson A \& Pluschke G (2007a) Genetic diversification of Neisseria meningitidis during waves of colonization and disease in the meningitis belt of sub-Saharan Africa. Vaccine 25(suppl 1): A18A23.

Leimkugel J, Hodgson A, Forgor AA et al. (2007b) Clonal waves of Neisseria colonisation and disease in the African meningitis belt: eight-year longitudinal study in northern Ghana. PLoS Med 4: e101.

Levin BR \& Cornejo OE (2009) The population and evolutionary dynamics of homologous gene recombination in bacterial populations. PLoS Genet 5: e1000601.

Maiden MC, Bygraves JA, Feil E et al. (1998) Multilocus sequence typing: a portable approach to the identification of clones within populations of pathogenic microorganisms. P Natl Acad Sci USA 95: 3140-3145.
Murphy GL, Connell TD, Barritt DS, Koomey M \& Cannon JG (1989) Phase variation of gonococcal protein II: regulation of gene expression by slipped-strand mispairing of a repetitive DNA sequence. Cell 56: 539-547.

Nicolas P, Norheim G, Garnotel E, Djibo S \& Caugant DA (2005) Molecular epidemiology of Neisseria meningitidis isolated in the African Meningitis Belt between 1988 and 2003 shows dominance of sequence type 5 (ST-5) and ST-11 complexes. J Clin Microbiol 43: 5129-5135.

Parkhill J, Achtman M, James KD et al. (2000) Complete DNA sequence of a serogroup A strain of Neisseria meningitidis Z2491. Nature 404: 502-506.

Russell JE, Jolley KA, Feavers IM, Maiden MC \& Suker J (2004) PorA variable regions of Neisseria meningitidis. Emerg Infect Dis 10: 674-678.

Sacchi CT, Lemos AP, Whitney AM, Solari CA, Brandt ME, Melles CE, Frasch CE \& Mayer LW (1998) Correlation between serological and sequencing analyses of the PorB outer membrane protein in the Neisseria meningitidis serotyping system. Clin Diagn Lab Immunol 5: 348-354.

Sié A, Pflüger V, Coulibaly B, Dangy JP, Kapaun A, Junghanss T, Pluschke G \& Leimkugel J (2008) ST2859 serogroup A meningococcal meningitis outbreak in Nouna Health District, Burkina Faso: a prospective study. Trop Med Int Health 13: 861-868.

Stephens DS, Greenwood B \& Brandtzaeg P (2007) Epidemic meningitis, meningococcaemia, and Neisseria meningitidis. Lancet 369: 2196-2210.

Tan LK, Carlone GM \& Borrow R (2010) Advances in the development of vaccines against Neisseria meningitidis. $N$ Engl J Med 362: 1511-1520.

Teyssou R \& Muros-Le RE (2007) Meningitis epidemics in Africa: a brief overview. Vaccine 25(suppl 1): A3-A7.

Thompson EA, Feavers IM \& Maiden MC (2003) Antigenic diversity of meningococcal enterobactin receptor FetA, a vaccine component. Microbiology 149: 1849-1858. 